\title{
A Generic Primary-control Model for Grid-forming Inverters: Towards Interoperable Operation \& Control
}

\author{
B. Johnson T.G. Roberts, O. Ajala, \& A. D. Domínguez-García \\ University of Washington University of Illinois at Urbana-Champaign University of Minnesota \\ brianbj@uw.edu \\ tgr2,ooajala2,aledan@illinois.edu \\ sdhople@umn.edu \\ D. Ramasubramanian, A. Tuohy \\ Electric Power Research Institute \\ D. Divan \\ Georgia Tech \\ dramasubramanian,atuohy@epri.com \\ B. Kroposki \\ National Renewable Energy Laboratory \\ benjamin.kroposki@nrel.gov
}

\begin{abstract}
This paper outlines an architectural vision centered around the notion of interoperability to integrate grid-forming inverter-based resources in large-scale grids. With the underlying principle of interoperability guiding developments, we focus on modeling the characteristics of droop, virtual synchronous machine, and virtual oscillator controls. Emphasis is placed on these three controllers since they are leading grid-forming control candidates and are likely to be commonplace as primary-control schemes in future systems. We show that these controllers can each be considered as instantiations of a more generic model and that all these controllers exhibit similar droop-like relations between pertinent terminal variables in steady state. This commonality between controllers gives interoperability among them such that automatic synchronization, power sharing, and voltage regulation can be achieved. Simulation results validate the models and demonstrate how the steady-state droop characteristics of these control methods can be aligned with the aid of the developed modeling paradigm.
\end{abstract}

\section{Introduction}

Current power-system operations and controls are primarily dictated by and tailored to the physical characteristics of synchronous machines. However, the structure and feasible functionalities of power

This work was authored in part by the National Renewable Energy Laboratory, operated by Alliance for Sustainable Energy, LLC, for the U.S. Department of Energy (DOE) under Contract No. DE-AC36-08GO28308. The views expressed in the article do not necessarily represent the views of the DOE or the U.S. Government. Support from the U.S. Department of Energy's Office of Energy Efficiency and Renewable Energy (EERE) under Solar Energy Technologies Office (SETO) Agreement Number EE0009025 is also gratefuly acknowledged. systems are rapidly evolving as more inverter-based resources (IBRs) are integrated into the grid [13]. It is now widely recognized that grid-forming (GFM) IBR technology will play a key role in at-scale integration alongside conventional assets [4]. With the anticipated shift to an increasing reliance on GFM-IBR-based generation and storage, the characteristics of power electronics inverters and their controls become paramount. Regardless of technology type (i.e., grid-following (GFL) or GFM), utilities need accurate models of inverters and their controllers to analyze stability and performance under a variety of conditions. However, it is challenging to acquire full visibility of inverter models and controllers, as vendors are understandably averse to disclosing IP-protected technology. Furthermore, increasing use of nonlinear control, dynamically varying system topologies and configurations, machine learning at the grid edge, and over-the-air software updates to millions of inverters from scores of vendors further compound challenges associated with scalable system-level analysis, performance verification, and stability certification [4].

One solution to facilitate the seamless integration of GFM IBRs alongside GFL IBRs and machines would be to develop vendor-agnostic guidelines that outline desired performance and describe pertinent phenomena at the point of grid interconnection. In this spirit, one could outline Interoperability Guidelines to promote the coordinated operation of a plurality of GFM technologies from multiple vendors while ensuring stability and reliability; and Functional Requirements to establish baseline GFM-IBR/plant/aggregation-level capabilities to comply with Interoperability Guidelines. This methodical categorization of unit- and system-level attributes can potentially facilitate protection of underlying intellectual property (IP) while enabling research, development, and demonstration activities that span multiple vendors. In fact, interoperability is 
at the heart of several consortia that leverage member cooperation to innovate while ensuring compliant products (e.g., the Bluetooth ${ }^{\circledR}$ and Wireless Power Charging consortia). In a future grid where potentially millions of inverters would have to operate to form and maintain the grid through a variety of corner cases, universal interoperability of GFM inverters deserves significant attention.

There are several facets to interoperability that have to be acknowledged in the modeling, analysis, operation, and control of GFM IBRs in future grids. In this paper, we focus on modeling, and establish a generic primary-control model that captures the dynamics of droop control [5], virtual synchronous machine (VSM) control [6], and dispatchable virtual oscillator control (dVOC) [7] under a single umbrella. The model is aligned with the notion of interoperability discussed above in the sense that different parameterizations of it boil down to the three different primary-control methods. From the outside-in, the specific implementations of the primary-control methods are not revealed. We anticipate such a model to streamline the characterization of pertinent stability and steady-state operational characteristics. In this spirit, we illustrate how the model facilitates parameterization to achieve similar steady-state terminal voltage and frequency under various loading conditions. Of practical importance, the similarities in their droop-like characteristics implies interoperability between all three control types with respect to communication-free synchronization, power-sharing, and voltage regulation. Since the generic primary-control model can be universally tailored to a wide variety of control methods, we anticipate it can facilitate standardization across modeling, control, and hardware development of grid-forming technologies. There have been previous attempts at unifying models for grid-forming inverters, see, e.g. [8-12]; however these have dominantly been limited in scope to pairs of control methods and not comprehensively sketched out parametric equivalences to the extent we attempt here.

The remainder of this paper is organized as follows. An overview of an envisioned system architecture emphasizing how interoperability will play a key role in integration of GFM IBRs is provided in Section 2. Zooming in on the main focus of this paper, i.e., interoperability in modeling, Section 3 discuses the three GFM primary-control models examined and outlines a generic primary control model that encapsulates all three as special cases. Simulation results to validate the modeling effort are provided in Section 4 . We conclude with a summary and outline a few directions for future work in Section 5.

\section{System Architecture}

In this section, we overview the system architecture that can be anticipated for integration of GFM IBRs across scales, and comment on how interoperability will play a key role in facilitating integration from a network level and the individual unit level.

\subsection{Overview of Architecture}

Figure 1 depicts the envisioned system architecture that builds on the prevailing architecture for power-system operations and control. It targets GFM integration alongside conventional assets/loads (not depicted) at levels approaching 100\% IBRs:

1. GFM technology will be interconnected and operated as plants (e.g., utility-scale PV/wind), aggregations (e.g., heterogeneous units at the distribution level capturing DER aggregations), or simply, individual GFM IBRs (to denote, e.g., units with significantly high capacity such as battery-storage systems).

2. Vendor-specific proprietary controls will coexist alongside interoperable primary control functions which facilitate the translation of system-level control guidelines in a vendorand application-agnostic manner.

3. Exchange of standardized signals through a suitable interface (labelled signal $I / O$ ) in a cyber-secure manner with the system operator will be essential to regulate frequency and voltages via secondary control and manage operations such as black-start.

4. To ensure scalability of the secondary-control architecture, distributed optimization and control algorithms within and across control areas (balancing areas) may be required.

The control architecture envisioned in Fig. 1 would be tailored based on system-specific characteristics and regulated by utilities and system operators. For instance, signals that are exchanged between GFM plants/IBRs/aggregations and operators would be standardized, and control implementations would build on prevailing methods for, e.g., frequency regulation via automatic generation control.

While there are several pieces to the architecture discussed above, the focus of this paper will be on on Point \#2. In particular, in what follows, we will outline an interoperable primary control model that ties together several popular GFM primary control methods published in the literature and deployed in practice. But first, we expound on notions of interoperability that are implied and necessary to realize a system architecture such as the one sketched in Fig. 1. 


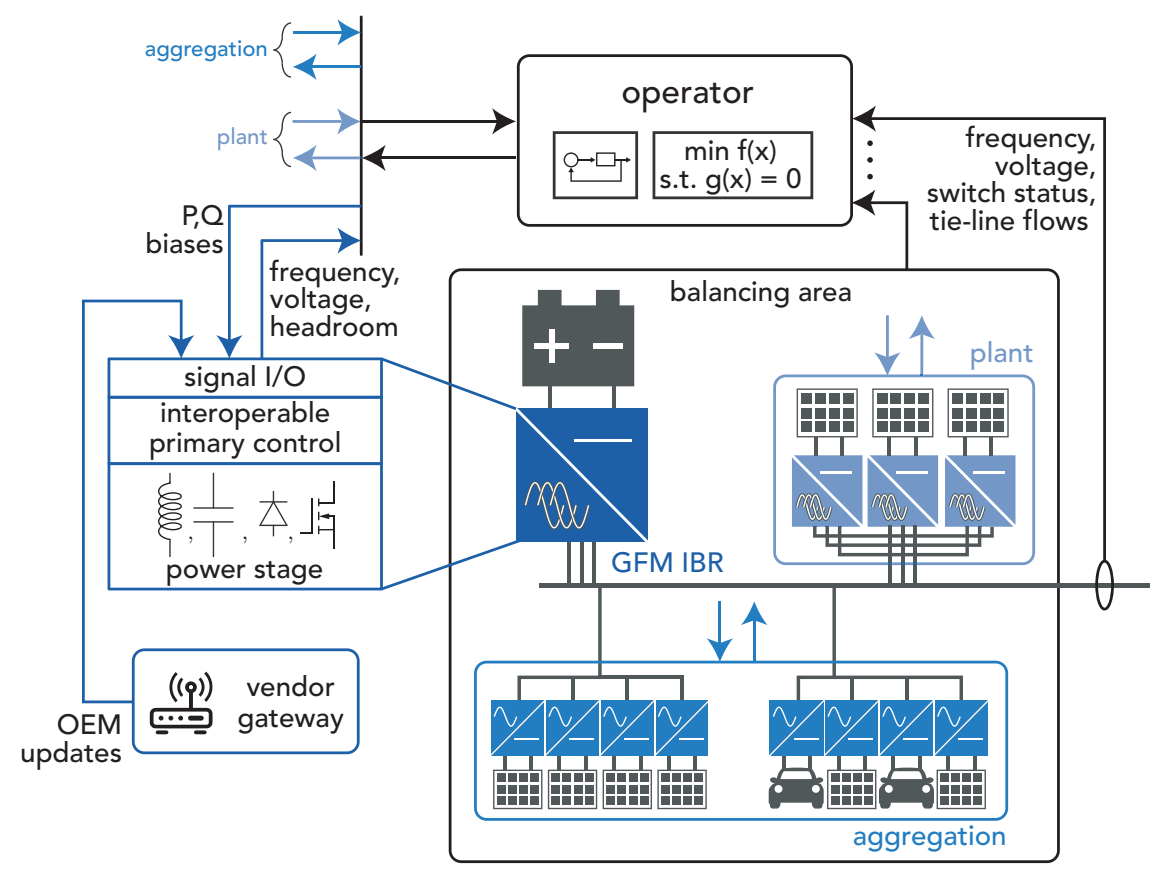

Figure 1: Envisioned system architecture depicting how GFM IBRs will be integrated across balancing areas. In this paper, we focus on outlining a generic interoperable primary control model that provides a common umbrella to examine three popular GFM primary control models: droop control, virtual synchronous machine control, and dispatchable virtual oscillator control.

\subsection{Interoperability as a Guiding Principle}

There are several notions of interoperability that spring to mind when considering the operation and control architecture in Fig. 1.

- Interoperable Primary Control. GFM IBRs should dynamically and autonomously adjust their active $(\mathrm{P})$ and reactive $(\mathrm{Q})$ power outputs in response to events (e.g., supply-demand imbalances, topology changes) that will manifest as frequency/voltage deviations. GFM IBRs are anticipated to share power in proportion to their capacities/ratings without external communication. Power sharing must be guaranteed while ensuring power balance in synchronous steady state and - to the extent possible-during transients on a per-balancing-area basis.

- Scalable Secondary Control. GFM IBRs should be compatible with system-level secondary-control architectures for frequency regulation, tie-line bias control, protection, and voltage control. GFM IBRs participating in system-level control will do so via a systematically engineered control stack that facilitates the translation of system-level operational requirements to proprietary controllers at the GFM-IBR/plant/aggregation level.

- Frequency \& Voltage Regulation Metrics. Primary- and secondary-control architectures should be tunable so they can satisfy a range of frequency- and voltage-regulation metrics with the full range of available control capability. The metrics should be quantifiable via available measurements at individual GFM-IBR/plant/aggregation or balancing-area levels.

- Real-time Control with Integrated Dynamic Protection. GFM IBRs should provide real-time controllability of instantaneous terminal voltage and frequency with integrated dynamic protection. Under nominal voltage and frequency conditions, these controllers will regulate GFM IBRs such that they can coexist in a heterogeneous mix of IBRs and machines. Through off-nominal excursions (including frequency swings, faults, and voltage sags) primary controllers will act to limit instantaneous electrical output.

- Signal I/O Interface. GFM IBRs will establish communication with system-level controllers via a standardized Signal I/O Interface. Anticipated signals that will have to be exchanged to target the full suite of secondary-control capability include (but are not limited to): apparent power nameplate rating, output-power maximum capacity, terminal voltage/frequency, and prevailing dispatch references/set-points for power and voltage. The Signal I/O Interface at the 
individual GFM-IBR/plant/aggregation level would communicate with the system's secondary-control architecture or plant/aggregation controller(s) as appropriate.

Broadly, we anticipate control and operations adhering to interoperable functionality discussed above to facilitate the coordinated operation of a plurality of GFM technologies from multiple vendors, while ensuring stability and reliability; and at the same time endowing individual IBRs/plants/aggregations with well defined functionality that can qualify them as GFM in a vendor-agnostic fashion. In what follows, we zoom in on the modeling and operation of the primary control layer discussed above.

\section{The GFM IBR Model}

In this section, we present a full-order averaged dynamical model for grid-forming inverters, as depicted in Fig. 2. Specifically, we consider three-phase inverters whose output voltage characteristics are determined by a droop, virtual synchronous machine (VSM), or dispatchable virtual oscillator control (dVOC) strategy. The electrical system of interest to us includes a three-phase inverter that is connected to an electrical power network bus through an $L C L$ filter, and the resulting full-order models are presented in a normalized per-unit form (see, e.g., [13, p. 75]). We begin this section by presenting mathematical models that describe the dynamics of droop, VSM, and dVOC, respectively. Afterwards, we present a generic primary control model, which is a generalized model with dynamical behavior, that under certain parametric assumptions, transforms to that of droop, VSM, or dVOC. Finally, we present a mathematical model that describes the dynamics of the $L C L$ filter which serves as a network interface for the grid-forming inverter.

\subsection{Reference-frame Transformation}

Let $\omega_{0}$ and $\omega(t)$, respectively, denote the nominal angular frequency and the angular frequency of a GFM IBR. Then, we denote the direct-quadrature reference frames rotating at frequency $\omega_{0}$ and $\omega_{i}(t)$ as $D Q$ and $d q$ reference frames, respectively. The $D Q$ reference frame represents a reference frame that is rotating in synchronism with the nominal angular velocity, commonly referred to as the synchronously rotating reference frame. On the other hand, the $d q$ reference frame represents a reference frame that may rotate at any angular velocity or remain stationary, commonly referred to as the arbitrary reference frame; all other direct-quadrature transformations can be obtained from the $d q$ reference frame by assigning the appropriate angular velocity [14, pp. 69-114]. Consider the balanced three-phase signal $f(t)=$ $\left[f_{a}(t), f_{b}(t), f_{c}(t)\right]^{\top}$. We denote $f^{\prime}(t)=\left[f_{D}(t)\right.$, $\left.f_{Q}(t)\right]^{\top}$ and $f^{\prime \prime}(t)=\left[f_{d}(t), f_{q}(t)\right]^{\top}$ to be the representations of the three-phase signal $f(t)$ in the $D Q$ and $d q$ reference frames, respectively. In other words, the single prime is used to denote a vector whose elements are state variables/signals that are expressed in the $D Q$ reference frame, and the double prime is used to denote a vector whose elements are state variables/signals that are expressed in the $d q$ reference frame. In developments that follow, balanced three-phase variables and companion signals will be represented in either the $D Q$ or $d q$ reference frame as appropriate. Define

$$
\delta(t)=\delta(0)+\int_{0}^{t}\left(\omega(x)-\omega_{0}\right) \mathrm{d} x
$$

where $\delta(0)$ denotes the value assumed by $\delta(t)$ at time $t=0$, and $\omega_{0}$ denotes the nominal frequency, e.g., $\omega_{0}=$ $2 \pi 60 \mathrm{rad} / \mathrm{s}$. Then, $f(t), f^{\prime}(t)$, and $f^{\prime \prime}(t)$ are related via

$$
f^{\prime \prime}(t)=\mathrm{T}_{2}(\delta(t)) f^{\prime}(t), \quad f^{\prime}(t)=\mathrm{T}_{1}\left(\omega_{0} t\right) f(t),
$$

where the $a b c$-to- $D Q$ transformation is realized with the transformation matrix

$\mathrm{T}_{1}(\alpha)=\frac{2}{3}\left[\begin{array}{ccc}\cos \alpha & \cos \left(\alpha-\frac{2 \pi}{3}\right) & \cos \left(\alpha+\frac{2 \pi}{3}\right) \\ -\sin \alpha & -\sin \left(\alpha-\frac{2 \pi}{3}\right) & -\sin \left(\alpha+\frac{2 \pi}{3}\right)\end{array}\right]$,

and the $D Q$-to- $d q$ transformation is realized with the rotation matrix

$$
\mathrm{T}_{2}(\alpha)=\left[\begin{array}{cc}
\cos \alpha & \sin \alpha \\
-\sin \alpha & \cos \alpha
\end{array}\right] .
$$

Additionally, the $D Q$-to- $a b c$ transformation is realized with the transformation matrix

$$
\mathrm{T}_{3}(\alpha)=\left[\begin{array}{cc}
\cos \alpha & -\sin \alpha \\
\cos \left(\alpha-\frac{2 \pi}{3}\right) & -\sin \left(\alpha-\frac{2 \pi}{3}\right) \\
\cos \left(\alpha+\frac{2 \pi}{3}\right) & -\sin \left(\alpha+\frac{2 \pi}{3}\right)
\end{array}\right],
$$

so that

$$
f(t)=\mathrm{T}_{3}\left(\omega_{0} t\right) f^{\prime}(t) .
$$

\subsection{Grid-forming Control Strategies}

We examine the dynamics of three grid-forming control strategies that have gained significant attention 


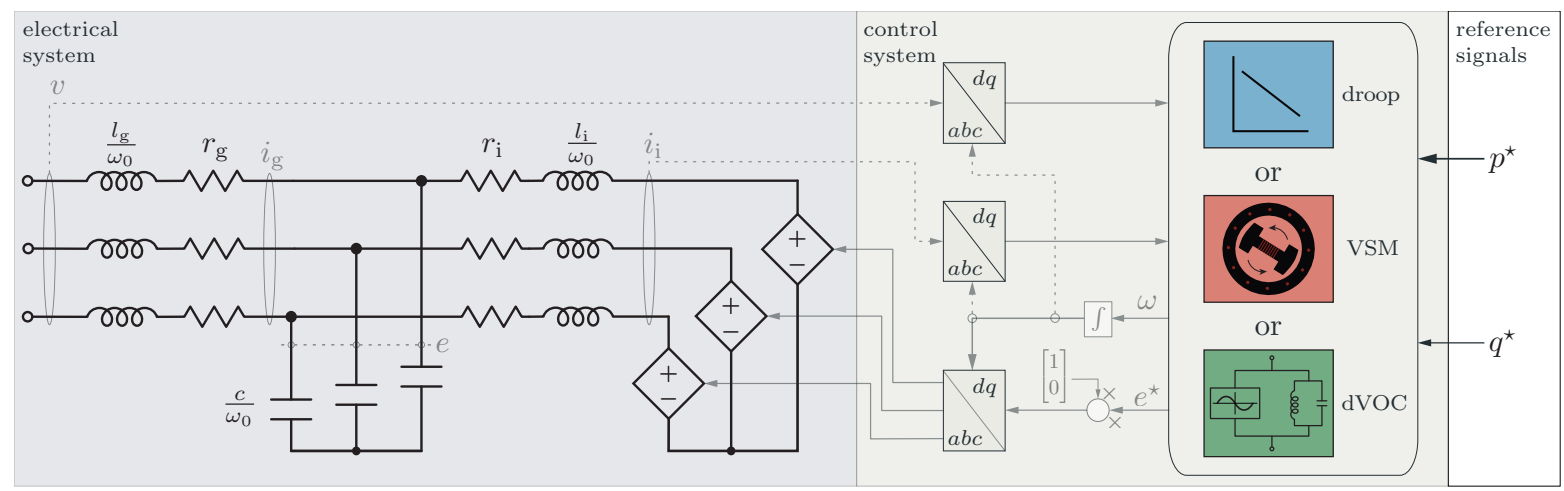

Figure 2: Schematic diagram of the averaged full-order GFM-inverter model with the reference signals, control system, and electrical system highlighted. The layers termed reference signals, control system, and electrical system, in the figure, correspond to the signal I/O block, the interoperable primary control block, and the power stage block in Fig. 1, respectively.

in the research community, namely: droop control, VSM control, and dVOC. Let $e_{0}$ denote the nominal voltage magnitude and $e^{\star}(t)$, the reference voltage magnitude of the three-phase inverter at time $t$, and let $i_{\mathrm{i}}(t)$ denote the three-phase inverter-side current of the $L C L$ filter at time $t$. Let $v(t)$ denote the three-phase voltage of the electrical network bus that the GFM inverter is connected to, let $p(t)$ and $q(t)$ denote the actual active and reactive power outputs of the three-phase inverter, respectively, and let $p_{\mathrm{m}}(t)$ and $q_{\mathrm{m}}(t)$ denote their measured values, all at time $t$. (See Fig. 2.) It follows that

$$
\begin{aligned}
& p(t)=e^{\star}(t) \mathbb{e}_{1}^{\top} \mathrm{T}_{2}(\delta(t)) \mathrm{T}_{1}\left(\omega_{0} t\right) i_{\mathrm{i}}(t), \\
& q(t)=e^{\star}(t) \mathbb{e}_{1}^{\top} \mathrm{T}_{2}\left(\delta(t)-\frac{\pi}{2}\right) \mathrm{T}_{1}\left(\omega_{0} t\right) i_{\mathrm{i}}(t),
\end{aligned}
$$

where $\mathbb{e}_{i}$ denotes the $2 \times 1$ standard basis vector with 1 in the $i$-th position. Let $p^{\star}$ and $q^{\star}$ denote the reference active- and reactive-power outputs of the three-phase inverter, respectively. GFM primary controllers typically have capability to yield tunable droop-like behavior in steady state. To this end, we will define $\psi \in[0,2 \pi)$ to be a rotation angle that, in steady state, determines how active power, reactive power, voltage, and frequency tradeoff.

Droop Control. In droop control, a cut-off frequency, $\omega_{c}$, is used to realize filtered versions of the output power, which are then used to generate values for the inverter angular frequency, $\omega(t)$, and the reference voltage magnitude, $e^{\star}(t)$. The inverter angular frequency is determined by penalizing output power deviations from reference values using the frequency droop coefficient $d_{\mathrm{f}}$, and adding the result to the nominal frequency $\omega_{0}$. In similar fashion, the reference voltage magnitude is determined by penalizing output power deviations from reference values using the voltage droop coefficient $d_{\mathrm{v}}$, and adding the result to the nominal voltage magnitude $e_{0}$. Accordingly, the droop control dynamics can be expressed as

$$
\begin{aligned}
\omega & =\omega_{0}+\frac{1}{d_{\mathrm{f}}} \mathbb{e}_{1}^{\top} \mathrm{T}_{2}\left(\psi-\frac{\pi}{2}\right)\left[\begin{array}{c}
p^{\star}-p_{\mathrm{m}} \\
q^{\star}-q_{\mathrm{m}}
\end{array}\right], \\
e^{\star} & =e_{0}+\frac{1}{d_{\mathrm{v}}} \mathbb{e}_{2}^{\top} \mathrm{T}_{2}\left(\psi-\frac{\pi}{2}\right)\left[\begin{array}{c}
p^{\star}-p_{\mathrm{m}} \\
q^{\star}-q_{\mathrm{m}}
\end{array}\right], \\
\frac{1}{\omega_{\mathrm{c}}}\left[\begin{array}{c}
\dot{p}_{\mathrm{m}} \\
\dot{q}_{\mathrm{m}}
\end{array}\right] & =-\left[\begin{array}{c}
p_{\mathrm{m}} \\
q_{\mathrm{m}}
\end{array}\right]+\left[\begin{array}{c}
p \\
q
\end{array}\right] .
\end{aligned}
$$

Steady-state Behavior. Setting the derivative terms in (5) to zero, and simplifying the resulting system of equations, we have that the steady-state models for angular frequency and voltage magnitude are given by:

$$
\begin{aligned}
\omega & =\omega_{0}+\frac{1}{d_{\mathrm{f}}} \mathbb{e}_{1}^{\top}\left[\begin{array}{cc}
\sin \psi & -\cos \psi \\
\cos \psi & \sin \psi
\end{array}\right]\left[\begin{array}{c}
p^{\star}-p \\
q^{\star}-q
\end{array}\right], \\
e^{\star} & =e_{0}+\frac{1}{d_{\mathrm{v}}} \mathbb{e}_{2}^{\top}\left[\begin{array}{cc}
\sin \psi & -\cos \psi \\
\cos \psi & \sin \psi
\end{array}\right]\left[\begin{array}{c}
p^{\star}-p \\
q^{\star}-q
\end{array}\right] .
\end{aligned}
$$

For all GFM control strategies discussed, we will see that $\psi=\frac{\pi}{2}$ imposes a strong correlation between active power and frequency, and between reactive power and voltage. Similarly, $\psi=0$ imposes a strong correlation between active power and voltage, and between reactive power and frequency. In the particular case of droop control examined here, for the special case when $\psi=\frac{\pi}{2}$, the steady-state model reduces to

$$
\omega=\omega_{0}+\frac{1}{d_{\mathrm{f}}}\left(p^{\star}-p\right), \quad e^{\star}=e_{0}+\frac{1}{d_{\mathrm{v}}}\left(q^{\star}-q\right),
$$


and for the special case when $\psi=0$, it reduces to

$$
\omega=\omega_{0}-\frac{1}{d_{\mathrm{f}}}\left(q^{\star}-q\right), \quad e^{\star}=e_{0}+\frac{1}{d_{\mathrm{v}}}\left(p^{\star}-p\right) .
$$

Virtual Synchronous Machine Control. In VSM control, a cut-off frequency, $\omega_{\mathrm{c}}$, is used to realize filtered versions of the output power, which are then used to generate values for the inverter angular frequency, $\omega(t)$, and the reference voltage magnitude, $e^{\star}(t)$. The inverter angular frequency is determined by penalizing output power deviations from reference values using the frequency droop coefficient $d_{\mathrm{f}}$, penalizing the mismatch between grid frequency and inverter frequency using the damping coefficient $d_{\mathrm{d}}$, adding both results to the nominal frequency $\omega_{0}$, and including an inertia term $m_{\mathrm{f}}$ to slow down the frequency response. In similar fashion, the reference voltage magnitude is determined by penalizing output power deviations from reference values using the voltage droop coefficient $d_{\mathrm{v}}$, and adding the result to the nominal voltage magnitude $e_{0}$. In VSM control, a phase-locked loop (PLL) is used to compute the frequency of the grid; we denote $\eta(t)$ and $\alpha(t)$ as the PLL's internal state variable and output phase, respectively. Accordingly, the VSM control dynamics can be expressed as

$$
\begin{aligned}
& \frac{m_{\mathrm{f}}}{d_{\mathrm{f}}} \dot{\omega}=-\omega+\omega_{0}+\frac{d_{\mathrm{d}}}{d_{\mathrm{f}}}\left(k_{\mathrm{P}} \dot{\eta}+\omega_{0} k_{\mathrm{I}} \eta\right) \\
&+\frac{1}{d_{\mathrm{f}}} \mathbb{e}_{1}^{\top} \mathrm{T}_{2}\left(\psi-\frac{\pi}{2}\right)\left[\begin{array}{c}
p^{\star}-p \\
q^{\star}-q_{\mathrm{m}}
\end{array}\right], \\
& e^{\star}= e_{0}+\frac{1}{d_{\mathrm{v}}} \mathbb{e}_{2}^{\top} \mathrm{T}_{2}\left(\psi-\frac{\pi}{2}\right)\left[\begin{array}{c}
p^{\star}-p \\
q^{\star}-q_{\mathrm{m}}
\end{array}\right], \\
& \frac{1}{\omega_{0}} \frac{\mathrm{d} \eta}{\mathrm{d} t}= \mathbb{e}_{2}^{\top} \mathrm{T}_{2}(\alpha) \mathrm{T}_{2}(\delta) \mathrm{T}_{1}\left(\omega_{0} t\right) v \\
& \frac{1}{\omega_{0}} \frac{\mathrm{d} \alpha}{\mathrm{d} t}=\frac{k_{\mathrm{P}}}{\omega_{0}} \dot{\eta}+k_{\mathrm{I}} \eta \\
& \frac{1}{\omega_{\mathrm{c}}} \dot{q}_{\mathrm{m}}=-q_{\mathrm{m}}+q
\end{aligned}
$$

where $k_{\mathrm{P}}$ and $k_{\mathrm{I}}$ denote the proportional and integral gains of the PLL, in per-unit, respectively.

Steady-state Behavior. Setting the derivative terms in (7) to zero, and simplifying the resulting system of equations, it follows that the steady state models for angular frequency and voltage magnitude in VSM control are described by (6), as in the case for droop control.

Dispatchable Virtual Oscillator Control. In dVOC, the inverter angular frequency is determined by penalizing output power deviations from reference values using the expression $\frac{\omega_{0} \kappa_{1}}{\left(e^{\star}\right)^{2}}$, where $\kappa_{1}$ denotes the synchronization gain of the controller, and adding the result to the nominal frequency $\omega_{0}$. In similar fashion, the reference voltage magnitude is determined by penalizing output power deviations from reference values using the expression $\frac{\kappa_{1}}{e^{\star}}$, penalizing voltage magnitude deviations from the nominal value using the expression $\kappa_{2} e^{\star}$, where $\kappa_{2}$ denotes the voltage-amplitude control gain, and using the sum of both results to generate values for the reference voltage magnitude. The dynamics of dVOC are:

$$
\begin{aligned}
\omega & =\omega_{0}+\frac{\omega_{0} \kappa_{1}}{\left(e^{\star}\right)^{2}} \mathbb{e}_{1}^{\top} \mathrm{T}_{2}\left(\psi-\frac{\pi}{2}\right)\left[\begin{array}{l}
p^{\star}-p \\
q^{\star}-q
\end{array}\right], \\
\frac{1}{\omega_{0} \kappa_{2}} \dot{e}^{\star} & =-\left(e^{\star}\right)^{3}+e_{0}^{2} e^{\star} \\
& +\frac{\kappa_{1}}{\kappa_{2} e^{\star}} \mathbb{e}_{2}^{\top} \mathrm{T}_{2}\left(\psi-\frac{\pi}{2}\right)\left[\begin{array}{c}
p^{\star}-p \\
q^{\star}-q
\end{array}\right] .
\end{aligned}
$$

Steady-state Behavior. Setting the derivative terms in (10) to zero, and simplifying the resulting system of equations, we have that the steady-state models for angular frequency and voltage magnitude are given by:

$$
\begin{aligned}
\omega & =\omega_{0}+\frac{\omega_{0} \kappa_{1}}{\left(e^{\star}\right)^{2}} \mathbb{e}_{1}^{\top}\left[\begin{array}{cc}
\sin \psi & -\cos \psi \\
\cos \psi & \sin \psi
\end{array}\right]\left[\begin{array}{l}
p^{\star}-p \\
q^{\star}-q
\end{array}\right], \\
0 & =-\left(e^{\star}\right)^{4}+\left(e_{0} e^{\star}\right)^{2} \\
& +\frac{\kappa_{1}}{\kappa_{2}} \mathbb{e}_{2}^{\top}\left[\begin{array}{cc}
\sin \psi & -\cos \psi \\
\cos \psi & \sin \psi
\end{array}\right]\left[\begin{array}{l}
p^{\star}-p \\
q^{\star}-q
\end{array}\right]
\end{aligned}
$$

with (9b) having the following roots:

$$
\begin{gathered}
\pm \frac{1}{\sqrt{2 \kappa_{2}}}\left(\kappa_{2} e_{0}^{2}-\left(\kappa_{2}^{2} e_{0}^{4}+4 \kappa_{2} \kappa_{1}\left(p^{\star}-p\right) \cos \psi\right.\right. \\
\left.\left.+4 \kappa_{2} \kappa_{1}\left(q^{\star}-q\right) \sin \psi\right)^{\frac{1}{2}}\right)^{\frac{1}{2}} \\
\pm \frac{1}{\sqrt{2 \kappa_{2}}}\left(\kappa_{2} e_{0}^{2}+\left(\kappa_{2}^{2} e_{0}^{4}+4 \kappa_{2} \kappa_{1}\left(p^{\star}-p\right) \cos \psi\right.\right. \\
\left.\left.+4 \kappa_{2} \kappa_{1}\left(q^{\star}-q\right) \sin \psi\right)^{\frac{1}{2}}\right)^{\frac{1}{2}}
\end{gathered}
$$

Note that

$$
\begin{aligned}
e^{\star} & =\frac{1}{\sqrt{2 \kappa_{2}}}\left(\kappa_{2} e_{0}^{2}+\left(\kappa_{2}^{2} e_{0}^{4}+4 \kappa_{2} \kappa_{1}\left(p^{\star}-p\right) \cos \psi\right.\right. \\
& \left.\left.+4 \kappa_{2} \kappa_{1}\left(q^{\star}-q\right) \sin \psi\right)^{\frac{1}{2}}\right)^{\frac{1}{2}}
\end{aligned}
$$


is the only root that produces real and non-negative values for $e^{\star}$ as the output power is varied across nominal values, under standard parametric assumptions. Accordingly, we will assume that the steady-state solution of $e^{\star}$ lies on a manifold described by this root.

For the special case when $\psi=\frac{\pi}{2}$, the steady-state model reduces to

$$
\begin{aligned}
\omega & =\omega_{0}+\frac{\omega_{0} \kappa_{1}}{\left(e^{\star}\right)^{2}}\left(p^{\star}-p\right), \\
e^{\star} & =\frac{1}{\sqrt{2 \kappa_{2}}}\left(\kappa_{2} e_{0}^{2}+\left(\kappa_{2}^{2} e_{0}^{4}+4 \kappa_{2} \kappa_{1}\left(q^{\star}-q\right)\right)^{\frac{1}{2}}\right)^{\frac{1}{2}},
\end{aligned}
$$

and for the special case when $\psi=0$, it reduces to

$$
\begin{aligned}
\omega & =\omega_{0}-\frac{\omega_{0} \kappa_{1}}{\left(e^{\star}\right)^{2}}\left(q^{\star}-q\right), \\
e^{\star} & =\frac{1}{\sqrt{2 \kappa_{2}}}\left(\kappa_{2} e_{0}^{2}+\left(\kappa_{2}^{2} e_{0}^{4}+4 \kappa_{2} \kappa_{1}\left(p^{\star}-p\right)\right)^{\frac{1}{2}}\right)^{\frac{1}{2}} .
\end{aligned}
$$

\subsection{The Generic Primary Control Model}

The underlying dynamics that govern the operation of the GFM inverter depend on the adopted control strategy, which could be a droop, VSM, or dVOC strategy. Accordingly, we define a generalized dynamical model whose equations, under certain parametric assumptions, transform to those of droop, VSM, or dVOC, and we refer to such a model as the generic primary control model. The dynamics of such a model are described by:

$$
\begin{aligned}
& \tau_{\mathrm{f}} \frac{\mathrm{d} \omega}{\mathrm{d} t}=-\omega+\omega_{0}+\kappa_{\mathrm{d}}\left(k_{\mathrm{P}} \dot{\eta}+\omega_{0} k_{\mathrm{I}} \eta\right) \\
& +\kappa_{\mathrm{f}} \mathbb{e}_{1}^{\top} \mathrm{T}_{2}\left(\psi-\frac{\pi}{2}\right)\left[\begin{array}{l}
p^{\star}-p_{\mathrm{m}} \\
q^{\star}-q_{\mathrm{m}}
\end{array}\right], \\
& \tau_{\mathrm{v}} \frac{\mathrm{d} e^{\star}}{\mathrm{d} t}=f_{\mathrm{v}}\left(e^{\star}\right)+\kappa_{\mathrm{v}} \mathbb{e}_{2}^{\top} \mathrm{T}_{2}\left(\psi-\frac{\pi}{2}\right)\left[\begin{array}{l}
p^{\star}-p_{\mathrm{m}} \\
q^{\star}-q_{\mathrm{m}}
\end{array}\right], \\
& \frac{1}{\omega_{0}} \frac{\mathrm{d} \eta}{\mathrm{d} t}=\mathbb{e}_{2}^{\top} \mathrm{T}_{2}(\alpha) \mathrm{T}_{2}(\delta) \mathrm{T}_{1}\left(\omega_{0} t\right) v \\
& \frac{1}{\omega_{0}} \frac{\mathrm{d} \alpha}{\mathrm{d} t}=\frac{k_{\mathrm{P}}}{\omega_{0}} \dot{\eta}+k_{\mathrm{I}} \eta, \\
& \tau_{\mathrm{p}}\left[\begin{array}{l}
\dot{p}_{\mathrm{m}} \\
\dot{q}_{\mathrm{m}}
\end{array}\right]=-\left[\begin{array}{l}
p_{\mathrm{m}} \\
q_{\mathrm{m}}
\end{array}\right]+\left[\begin{array}{l}
p \\
q
\end{array}\right],
\end{aligned}
$$

where $\tau_{\mathrm{f}}, \tau_{\mathrm{v}}, \tau_{\mathrm{p}}, \kappa_{\mathrm{d}}, \kappa_{\mathrm{f}}, \kappa_{\mathrm{v}}$, and $f_{\mathrm{v}}\left(e^{\star}\right)$ take different forms depending on the GFM control strategy, as described in Table 1.

Steady-state Behavior. Setting the derivative terms in (13) to zero, and simplifying the resulting system of equations, we have that the steady-state models for angular frequency and voltage magnitude are given by:

$$
\begin{aligned}
& \omega=\omega_{0}+\kappa_{\mathrm{f}} \mathbb{E}_{1}^{\top}\left[\begin{array}{cc}
\sin \psi & -\cos \psi \\
\cos \psi & \sin \psi
\end{array}\right]\left[\begin{array}{l}
p^{\star}-p \\
q^{\star}-q
\end{array}\right], \\
& f_{\mathrm{v}}\left(e^{\star}\right)=-\kappa_{\mathrm{v}} \mathbb{e}_{2}^{\top}\left[\begin{array}{cc}
\sin \psi & -\cos \psi \\
\cos \psi & \sin \psi
\end{array}\right]\left[\begin{array}{l}
p^{\star}-p \\
q^{\star}-q
\end{array}\right] .
\end{aligned}
$$

For the special case when $\psi=\frac{\pi}{2}$, the steady-state model reduces to

$$
\omega=\omega_{0}+\kappa_{\mathrm{f}}\left(p^{\star}-p\right), \quad 0=f_{\mathrm{v}}\left(e^{\star}\right)+\kappa_{\mathrm{v}}\left(q^{\star}-q\right),
$$

and for the special case when $\psi=0$, it reduces to

$$
\omega=\omega_{0}-\kappa_{\mathrm{f}}\left(q^{\star}-q\right), \quad 0=f_{\mathrm{v}}\left(e^{\star}\right)+\kappa_{\mathrm{v}}\left(p^{\star}-p\right) .
$$

Steady-state Frequency Droop. The frequency droop coefficient associated with the generic primary control model can be set according to the preferred steady-state behavior of the GFM inverter. For example, consider the case when $\psi=\frac{\pi}{2}$ and $p^{\star}=0$. If the desired steady-state behavior is such that a 1 per-unit increase in output power $p$ results in a $x \%$ frequency deviation from nominal, then this can be achieved by setting $\kappa_{\mathrm{f}}=$ $\frac{x}{100} \omega_{0}$. As a specific example, a $5 \%$ frequency droop is realized by setting $\kappa_{\mathrm{f}}=0.05 \omega_{0}$.

Steady-state Voltage Droop. The voltage droop coefficient associated with the generic primary control model can be set according to the preferred steady-state behavior of the GFM inverter. However, the procedure for choosing the voltage droop coefficient varies depending on the GFM control strategy. For example, consider a droop or VSM strategy with $\psi=\frac{\pi}{2}$ and $q^{\star}=0$. If the desired steady-state behavior is such that a 1 per-unit increase in output power $q$ results in a $x \%$ voltage deviation from nominal, then this can be achieved by setting $\kappa_{\mathrm{v}}=\frac{x}{100} e_{0}$. However, the same behavior is achieved in $\mathrm{dVOC}$ by setting

$$
\kappa_{\mathrm{v}}=\frac{100^{4}-\left(2(100-x)^{2}-100^{2}\right)^{2}}{4 \times 100^{4}} e_{0}^{4} .
$$

As a specific example, a $2 \%$ voltage droop is realized in droop or VSM by setting $\kappa_{\mathrm{V}}=0.02 e_{0}$, but it is realized in dVOC by setting $\kappa_{\mathrm{V}}=0.038 e_{0}^{4}{ }^{1}$

\footnotetext{
${ }^{1}$ The expression for $\kappa_{\mathrm{V}}$, in a dVOC strategy, is derived from (11) by replacing $e^{\star}$ with $\frac{100-x}{100}$ and solving for $\frac{\kappa_{1}}{\kappa_{2}}$; this is due to the fact that $\kappa_{\mathrm{v}}=\frac{\kappa_{1}}{\kappa_{2}}$ in dVOC.
} 
TABLE 1

PARAMETRIC ASSUMPTIONS UNDER WHICH THE GENERIC PRIMARY CONTROL MODEL TRANSFORMS TO DROOP CONTROL, VSM CONTROL, AND DVOC.

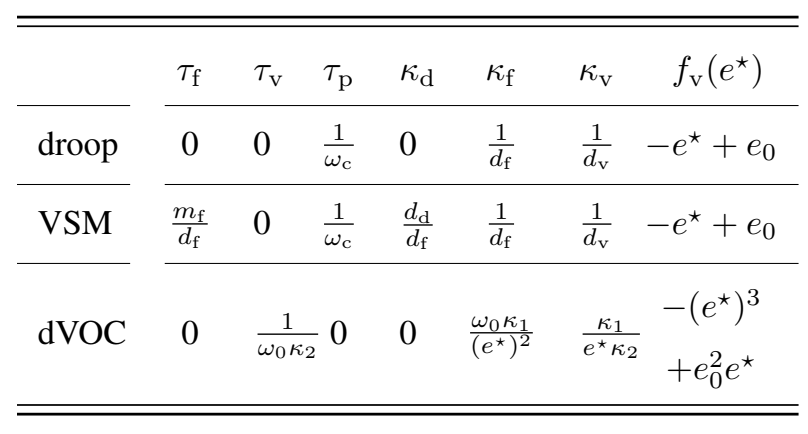

\subsection{Network interface for GFM models}

Consider a GFM inverter that is connected to an electrical network bus through an $L C L$ filter. Let $i_{\mathrm{i}}(t)$ $\left(i_{\mathrm{g}}(t)\right)$ denote the three-phase inverter-side (grid-side) current of the $L C L$ filter, let $e(t)$ denote the three-phase voltage across the filter's capacitor, and let $v$ denote the three-phase voltage of the electrical network bus that the GFM inverter is connected to. Then the dynamics of the $L C L$ filter are described by

$$
\begin{aligned}
\frac{l_{\mathrm{i}}}{\omega_{0}} \frac{\mathrm{d} i_{\mathrm{i}}}{\mathrm{d} t} & =-r_{\mathrm{i}} i_{\mathrm{i}}+\mathrm{T}_{3}\left(\omega_{0} t\right) \mathrm{T}_{2}(-\delta) \mathbb{e}_{1} e^{\star}-e, \\
\frac{c}{\omega_{0}} \frac{\mathrm{d} e}{\mathrm{~d} t} & =i_{\mathrm{i}}-i_{\mathrm{g}} \\
\frac{l_{\mathrm{g}}}{\omega_{0}} \frac{\mathrm{d} i_{\mathrm{g}}}{\mathrm{d} t} & =-r_{\mathrm{g}} i_{\mathrm{g}}+e-v
\end{aligned}
$$

where $r_{\mathrm{i}}$ and $l_{\mathrm{i}}$ denote the inverter-side resistance and inductance, respectively, $r_{\mathrm{g}}$ and $l_{\mathrm{g}}$ denote the grid-side resistance and inductance, respectively, and $c$ denotes the filter capacitance.

\section{Simulation Case Study}

In this section, we describe the test system that is used to develop numerical results for the different grid-forming control technologies. We begin by describing the test scenarios considered, and following that, describe the resulting numerical results.

\subsection{Setup}

The goal of these simulations is to compare the steady-state behavior of the droop, VSM, and dVOC control strategies, by observing, for each scheme, the steady-state relationships between frequency, voltage, and output power deviations from reference values. The corresponding results for droop control are developed by using (6), and considering two cases, i.e., $\psi=\frac{\pi}{2}$, which allows for $p^{\star}$ tracking, and $\psi=0$, which allows for $q^{\star}$ tracking. Given that the steady-state expressions for droop and VSM are exactly the same, their results are equivalent. On the other hand, the results for dVOC are developed using (9a) and (9b), with the real non-negative root for $e^{\star}$ specified in (11). Specifying a $0.33 \%$ frequency droop and a $4 \%$ voltage droop for the test system, values for $d_{\mathrm{f}}, d_{\mathrm{v}}, \kappa_{1}$, and $\kappa_{2}$ are selected according to the procedures outlined in Section 3.3 (see Table 2 for system parameter values).

\subsection{Results}

Figure 3 illustrates steady-state relationships between inverter frequency, reference-voltage magnitude, and the output-power deviations from reference values for droop, VSM, and dVOC. Surfaces colored red-yellow correspond to dVOC. The blue-purple color denotes both droop and VSM (since, as the analysis above indicates, their responses are identical). Broadly, we see that with appropriate tuning aided by the insight offered by the developed generic model, we can engineer nearly the same steady-state response for the different primary-control strategies. Indeed, steady-state behavior can be differentiated universally, i.e., across all three methods, based on values of $\psi$. We overview this next.

When $\psi=\frac{\pi}{2}$, we observe that there is a strong

TABLE 2

Simulation PARAMETERS For CASE StUdies.

\begin{tabular}{lllcl}
\hline \hline \multirow{2}{*}{ Symbol } & & Description & Value & Units \\
\cline { 1 - 3 } \cline { 3 - 5 }$e_{0}$ & & Nominal voltage magnitude & 1 & $\mathrm{pu}$ \\
\hline$\omega_{0}$ & Nominal frequency & $2 \pi 60$ & $\mathrm{rad} / \mathrm{s}$ \\
\hline$\omega_{\mathrm{c}}$ & Cut-off frequency & $2 \pi 20$ & $\mathrm{rad} / \mathrm{s}$ \\
\hline$l_{\mathrm{i}}$ & Filter inductance & 0.02 & $\mathrm{pu}$ \\
\hline$r_{\mathrm{i}}$ & Filter resistance & 0.014 & $\mathrm{pu}$ \\
\hline$c$ & Filter capacitance & 0.11 & $\mathrm{pu}$ \\
\hline$l_{\mathrm{g}}$ & Line inductance & 0.02 & $\mathrm{pu}$ \\
\hline$r_{\mathrm{g}}$ & Line resistance & 0.014 & $\mathrm{pu}$ \\
\hline$d_{\mathrm{v}}$ & Frequency droop coefficient & 0.8038 & $\mathrm{~s} / \mathrm{rad}$ \\
\hline$\kappa_{1}$ & Voltage droop coefficient & 25 & $\mathrm{pu}$ \\
\hline$\kappa_{2}$ & Synchronization gain & 0.0033 & $\mathrm{pu}$ \\
\hline$k_{\mathrm{P}}$ & Voltage-amplitude control gain & 0.0457 & $\mathrm{pu}$ \\
\hline$k_{\mathrm{I}}$ & Proportional gain of PLL & 50 & $\mathrm{pu}$ \\
\hline \hline & Integral gain of PLL & 1 & $\mathrm{pu}$ \\
\hline
\end{tabular}




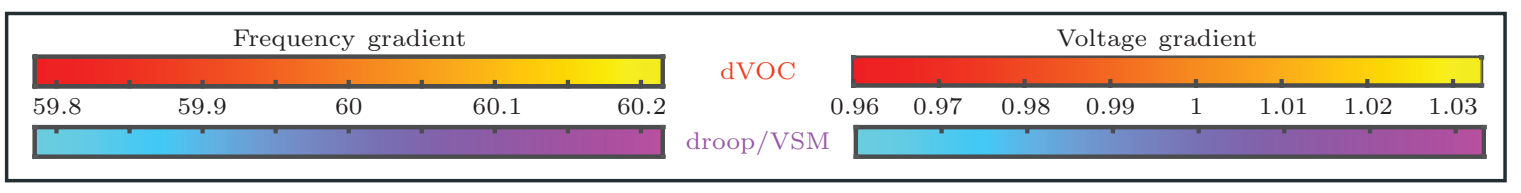
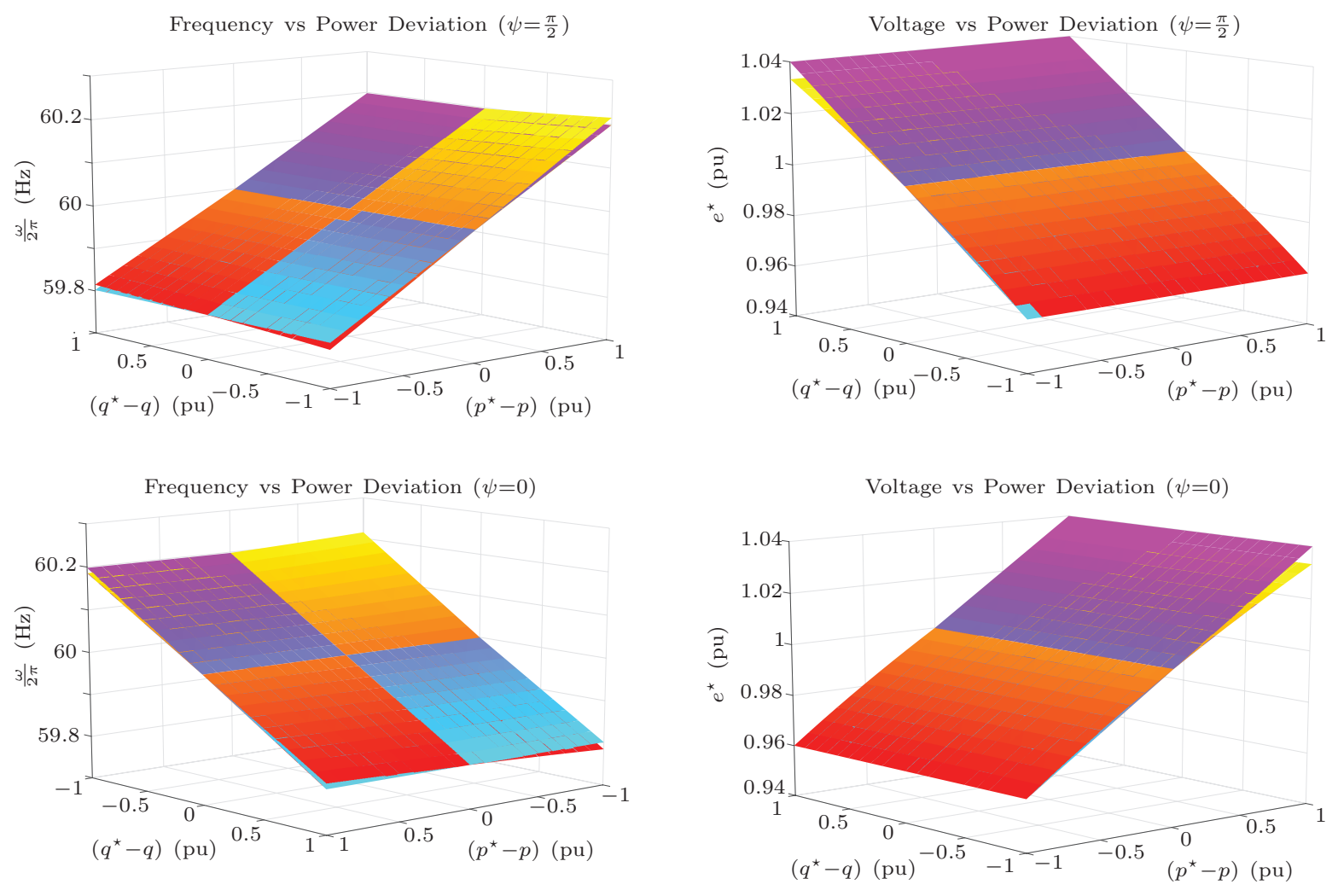

Figure 3: The steady-state relationships between the inverter's frequency, reference voltage magnitude, and the output-power deviations from reference values are depicted. Results are depicted for droop, VSM, and dVOC, with $\psi=\frac{\pi}{2}$ or $\psi=0$. Surfaces colored red-yellow correspond to dVOC, while the blue-purple color denotes both droop and VSM since their responses are identical. On the left hand side are frequency vs. power deviation plots; here, the $\mathrm{dVOC}$ is non-linear but close to the linear response of droop/VSM for the range considered. The graph shows the linear droop/VSM plane intersecting at both $\left(q^{\star}-q\right)=0$ and $\left(p^{\star}-p\right)=0$. The plots on the right hand side depict voltage vs. power deviation; here, when $\psi=\frac{\pi}{2}(\psi=0)$ the linear droop/VSM plane cut through the dVOC plane at $\left(q^{\star}-q\right)=0\left(\left(p^{\star}-p\right)=0\right)$. A key observation from these results is that nearly the same steady-state response can be achieved by the droop, VSM, and dVOC control strategy.

correlation between frequency $\omega$ and active power deviation $p^{\star}-p$, as well as between voltage reference $e^{\star}$ and reactive power deviation $q^{\star}-q$. Specifically, in all three control strategies, the frequency (voltage reference) increases as the active (reactive) power deviation increases, and vice versa. This implies that as the inverter injects more active (reactive) power into the bus, the frequency (voltage reference) decreases to compensate for the increasing active (reactive) power injection. It is interesting to note that while the steady state plots for dVOC are not quite linear, its droop characteristics when $\left(q^{\star}-q\right) \in[-1,1]$ and $\left(p^{\star}-p\right) \in$ $[-1,1]$ are very similar to those of droop and VSM, which have a linear steady state droop behavior. The steady state plots intersect when $\left(q^{\star}-q\right)=0$ and $e^{\star}=1$.

When $\psi=0$, we observe that there is a strong correlation between frequency $\omega$ and reactive power deviation $q^{\star}-q$, as well as between voltage reference $e^{\star}$ and active power deviation $p^{\star}-p$. This is the opposite of the correlations observed for the case when $\psi=\frac{\pi}{2}$. By switching from $\psi=\frac{\pi}{2}$ to $\psi=0$, the relationship 
between $e^{\star}$ vs $\left(q^{\star}-q\right)$ is translated to $e^{\star}$ vs $\left(p^{\star}-p\right)$, and the relationship between $\omega$ and $\left(p^{\star}-p\right)$ is translated to $\omega$ vs $\left(q-q^{\star}\right)$.

\section{Conclusions and Future Work}

In this paper, we examined models for grid-forming inverters under the lens of interoperability with a focus on the primary-control dynamics. Particularly, we developed a generic primary control model, that under specific parametric assumptions, yields the dynamics of three popular grid-forming primary control methods: droop control, virtual synchronous machine control, and dispatchable virtual oscillator control. Design strategies to align the steady-state operation of the methods were developed and validated with simulations. Broadly, interoperable models of the form we outline will be critical in standardizing simulation efforts across packages as well as in control synthesis.

Future work includes examining behavior under large disturbances and post-fault conditions with particular emphasis on the accuracy of the model(s) as GFM inverters operate at (or around) limits. Furthermore, for broad adoption, it would be critical to integrate such interoperable models in standard simulation packages and leverage them for secondary control with a focus on abstracting inner control loops and implementation details in individual grid-forming inverters. Case studies can also be extended to more complex network settings involving a mix of grid-forming inverters, grid-following inverters, and synchronous generators with varied power ratings.

\section{References}

[1] J. A. Taylor, S. V. Dhople, and D. S. Callaway, "Power systems without fuel," Renewable and Sustainable Energy Reviews, vol. 57, pp. 1322-1336, May 2016.

[2] B. Kroposki, B. Johnson, Y. Zhang, V. Gevorgian, P. Denholm, B.-M. Hodge, and B. Hannegan, "Achieving a $100 \%$ renewable grid: Operating electric power systems with extremely high levels of variable renewable energy," IEEE Power and Energy Magazine, vol. 15, no. 2, pp. 61-73, 2017.

[3] D. Ramasubramanian, P. Pourbeik, E. Farantatos, and A. Gaikwad, "Simulation of $100 \%$ inverter-based resource grids with positive sequence modeling," IEEE Electrification Magazine, vol. 9, no. 2, pp. 62-71, 2021.

[4] Y. Lin, J. H. Eto, B. B. Johnson, J. D. Flicker, R. H. Lasseter, H. N. V. Pico, G.-S. Seo, B. J. Pierre, and A. Ellis, "Research roadmap on grid-forming inverters," tech. rep., National Renewable Energy Laboratory, NREL/TP-5D00-73476, 2020.

[5] M. C. Chandorkar, D. M. Divan, and R. Adapa, "Control of parallel connected inverters in standalone AC supply systems," IEEE Transactions on Industry Applications, vol. 29, pp. 136-143, Jan. 1993.
[6] S. D'Arco, J. Suul, and O. Fosso, "A virtual synchronous machine implementation for distributed control of power converters in smartgrids," Electric Power Systems Research, vol. 122, pp. 180 - 197, 2015.

[7] M. Lu, S. Dutta, V. Purba, S. Dhople, and B. Johnson, "A grid-compatible virtual oscillator controller: Analysis and design," in 2019 IEEE Energy Conversion Congress and Exposition (ECCE), pp. 2643-2649, Sep. 2019.

[8] S. D'Arco and J. A. Suul, "Equivalence of virtual synchronous machines and frequency-droops for converter-based microgrids," IEEE Transactions on Smart Grid, vol. 5, no. 1, pp. 394-395, 2014.

[9] M. Sinha, S. Dhople, B. Johnson, N. Ainsworth, and F. Dörfler, "Nonlinear supersets to droop control," in 2015 IEEE 16th Workshop on Control and Modeling for Power Electronics (COMPEL), pp. 1-6, 2015.

[10] M. Sinha, F. Dörfler, B. B. Johnson, and S. V. Dhople, "Uncovering Droop Control Laws Embedded Within the Nonlinear Dynamics of Van der Pol Oscillators," IEEE Transactions on Control of Network Systems, vol. 4, no. 2, pp. 347-358, 2017.

[11] A. Tayyebi, D. Groß, A. Anta, F. Kupzog, and F. Dörfler, "Frequency stability of synchronous machines and grid-forming power converters," IEEE Journal of Emerging and Selected Topics in Power Electronics, vol. 8, no. 2, pp. 1004-1018, 2020.

[12] B. Johnson, M. Rodriguez, M. Sinha, and S. Dhople, "Comparison of virtual oscillator and droop control," in 2017 IEEE 18th Workshop on Control and Modeling for Power Electronics (COMPEL), pp. 1-6, 2017.

[13] P. Kundur, N. J. Balu, and M. G. Lauby, Power System Stability and Control. McGraw-Hill, 1994.

[14] A. Yazdani and R. Iravani, Voltage-Sourced Converters in Power Systems. Wiley, Jan. 2010. 